\title{
Justicia y eficacia \\ Una lectura del pensamiento político humeano
}

\author{
Eduardo Óscar Charpenel Elorduy \\ Facultad de Filosofía \\ Universidad Nacional Autónoma de México \\ echarpenel@gmail.com
}

\section{Introducción}

Uno de los puntos neurálgicos para entender el pensamiento moral y político de David Hume es, sin lugar a dudas, la distinción que realiza el filósofo escocés entre virtudes naturales y artificiales. Se trata de una distinción que lo ubica en una posición muy particular con respecto a otros filósofos que lo antecedieron pues, por un lado, lo distancia de posturas que buscaban el fundamento de la moral exclusivamente en los sentimientos (Shaftesbury, Hutcheson) y, por otro, lo aleja de aquellos que negaban toda forma de moralidad fuera de los límites de un Estado soberano (Hobbes). En la polémica por determinar las bases de la moral, Hume desarrolla una posición intermedia que articula los principios de estos dos bandos, logrando crear una teoría que tematiza los rasgos que caracterizan a la convivencia humana virtuosa en cualquiera de sus distintos niveles. En este ensayo, estudiaré principalmente cómo es que, a juicio de Hume, los seres humanos razonamos en términos de justicia. Abordar esta pregunta es algo de suma importancia para comprender de forma adecuada cómo concibe Hume la vida en comunidad dentro de su filosofía. Después de precisar en qué radica el carácter específicamente artificial de la justicia, analizaré cuáles son a su juicio los principales fundamentos del Estado y con ello mostraré qué factores determinan la conformación de los cuerpos políticos y la adhesión de los ciudadanos a los mismos. 
Este último punto será clave para poder situar la originalidad de la filosofía política humeana en un contexto dominado por las tradiciones contractualistas.

\section{El origen de la distinción: algunas consideraciones sobre las virtudes naturales}

Revisemos, en primer lugar, aquella distinción que subyace a todo el pensamiento moral y político de Hume. A su juicio, la naturaleza ha implantado en el ser humano unos sentimientos que hacen que éste apruebe o rechace las acciones que realizan sus congéneres. En este sentido, la experiencia moral más elemental consiste en experimentar atracción o repulsión por la conducta que observamos en los demás. El origen de esos sentimientos de aprobación o rechazo, de acuerdo con Hume, es la observación directa de las virtudes y los vicios de las otras personas ${ }^{1}$. Estas cualidades positivas o negativas son lo primero que evaluamos en un sujeto cuando nos pronunciamos moralmente sobre él. A diferencia de Aristóteles, por ejemplo, que caracterizaba a la virtud como un justo medio entre dos extremos viciosos, Hume concibe a la virtud como la cualidad en el obrar que provoca que nosotros alabemos o censuremos espontáneamente a alguien ${ }^{2}$. Lo que resulta «natural» de estas virtudes es que no han sido inventadas o creadas por la sociedad y que no estamos determinados por nuestro contexto histórico o cultural a ejecutarlas o a responder de forma empática cuando observamos

${ }^{1}$ Brand encuentra dos momentos distintos en la evaluación moral de la teoría humeana: «El proceso de simpatizar con otro tiene dos momentos, uno cognitivo y otro afectivo. El primer momento explica cómo adquirimos nosotros una idea del estado pasional del otro. El segundo momento comprende la transformación de esta idea en una impresión, de modo que lleguemos a sentir la emoción que creemos que el otro está sintiendo», en: Walter BRAND, Hume's Theory of Moral Judgment, Dodrecht: Kluwer Academic Publishers 1992, p. 45. Las citas del inglés de las obras serán mías a menos que señale lo contrario.

2 Cf. Rachel COHON, «Hume's Artificial and Natural Virtues», en: The Blackwell Guide to Hume's Treatise, Oxford: Blackwell 2006 p. 257. En dicha comparación, Cohon resalta de manera acertada otro punto importante: la práctica de la virtud para Aristóteles está orientada hacia la consecución de la eudaimonía. Hume no busca en ningún momento determinar cómo la virtud perfecciona a quien la realiza. 
que alguien las practica. Si bien somos capaces de cultivar de mejor o peor manera las disposiciones éticas que la naturaleza ha implantado en nosotros, lo principal de éstas es que tienen su fundamento en la constitución misma del ser humano. De ello se sigue, por ejemplo, que una persona censure el egoísmo o alabe la generosidad de otra persona no porque su educación o entorno la condicionen absolutamente a reaccionar así, sino porque el temperamento humano está inclinado a reaccionar de esta manera cuando uno contempla las acciones de alguien que sólo vela por su propio bienestar, o bien, cuando uno observa la conducta de alguien que antepone el bienestar de los demás al propio. Hume define a la simpatía como la «propensión de recibir los sentimientos y las inclinaciones de los otros a través de la comunicación» ${ }^{3}$. Resulta evidente que en esta definición de simpatía se evidencia el asociacionismo de la teoría del conocimiento humeana según la cual tendemos a vincular ideas e impresiones semejantes de las cosas a través del hábito. No es el lugar para entrar en los pormenores de dicha teoría: baste decir, en este contexto, que una persona puede sentir empatía por otra persona que actúa de forma benevolente en la medida en que es capaz de reconocer en sí misma la capacidad de actuar motivado por pasiones nobles parecidas. Es evidente para Hume que «cuando alabamos cualquier acción únicamente evaluamos los motivos que las produjeron, y consideramos las acciones como signos o indicadores de ciertos principios de la mente o del temperamento» ${ }^{4}$. De manera análoga, una persona es capaz de sentir empatía por otra persona en desgracia en virtud de la pasión compasiva que tal situación despierta en él, una pasión que está acompañada de una asociación de ideas que le permite al sujeto colocarse imaginariamente en un estado semejante de privaciones y carencias.

Existen varias pasiones y sentimientos que hacen que los individuos actúen de forma benevolente y que, por lo mismo, hacen que nosotros como espectadores las consideremos como buenas.

${ }^{3}$ David HUME, A Treatise of Human Nature, Oxford: Oxford University Press 2000, p. 316. (Utilizaré la paginación crítica de Selby Bigge tanto para esta obra como para la Investigación sobre los principios de la moral).

${ }^{4}$ HUME, A Treatise..., p. 477. 
La benevolencia, por su parte, es la más elevada de las cualidades humanas, pues a juicio de Hume no existen otras «que tengan más derecho a la buena voluntad y a la aprobación general de la humanidad, la amistad y la gratitud, el afecto natural y el espíritu público, o cualquiera otra que proceda de una tierna simpatía para con los demás y de una preocupación generosa por nuestra clase y especie» ${ }^{5}$. En la Investigación, la representación de la benevolencia como algo admirable está íntimamente ligada al concepto de utilidad. No se trata sólo de una utilidad para el propio agente, sino de una utilidad que también conlleva felicidad y satisfacción para los otros miembros de la sociedad. La benevolencia es una virtud que consolida la relación con los padres, refuerza la autoridad con los hijos y, en general, fomenta la disposición de ayudar que tiene esta persona en todos los sectores y ámbitos. La utilidad que se deriva de las virtudes sociales constituye, al menos, una parte de su mérito, y es según Hume «una fuente de esa aprobación y estima que universalmente se les presta» ${ }^{6}$.

Como se aprecia a primera vista, el ejercicio de tales virtudes supone una repercusión benéfica dentro de una comunidad. Su origen subjetivo en las pasiones y los sentimientos no constituye nunca un obstáculo para que las virtudes incidan de manera positiva en el entorno social. Sin embargo, el que la benevolencia se encuentre fundada en las pasiones humanas sí determina que las ejerzamos de manera parcial7. En otras palabras, somos más proclives a ser bondadosos con personas más allegadas a nosotros. Quizás esto no constituye en sí mismo algo reprochable. Es perfectamente lógico que una madre muestre una marcada preferencia por su hijo que por los hijos de otras mujeres; más aún, con justa razón se le podría reclamar a la madre que no actuara $a^{\prime} i^{8}$. Lo que tal disposición refleja es que la práctica de estas virtudes no es uniforme entre los seres humanos. Somos más propensos a ejercer estas virtudes

${ }^{5}$ David HUME, Investigación sobre los principios de la moral, trad. Gerardo López Sastre, Madrid: Tecnos 2007, p. 187.

${ }^{6}$ HUME, Investigación sobre..., p. 179.

${ }^{7}$ HUME, A Treatise..., 488-489 pp.

${ }^{8}$ Cf. COHON, «Hume's Aritificial...», p. 289. 
cuando tratamos a personas cuyos vínculos con nosotros son más íntimos y firmes.

\section{La justicia como virtud arquitectónica de la sociedad}

Aunque no escatima elogios para las virtudes naturales, Hume se da cuenta de que éstas resultan insuficientes para fundar sólidamente la vida en comunidad, pues sólo en ella el hombre puede sobrevivir y obtener la mayor cantidad de beneficios para su propia existencia. De ahí que afirme que el hombre tenga que echar mano de su razón - y ya no de su sentimiento- para delinear las estructuras de una comunidad funcional. El ejercicio de la razón, en este ámbito, constituye para Hume una virtud esencialmente política. Por lo mismo, se trata de una virtud «artificial» en más de un sentido. La justicia es una virtud que debemos de practicar con aquellos que no se encuentran en nuestro entorno más inmediato - es decir, aquellos con quienes en principio nos comportarnos virtuosamente - y con quienes no tenemos lazos afectivos fuertes. Además, no es propiamente una pasión como la simpatía o la benevolencia la que nos lleva a ejecutarla o a practicarla, sino el cálculo que, por ejemplo, contempla la solución a problemas de escasez y de insuficiencia de bienes, y así poder generar la mayor utilidad posible. En palabras de Stroud, «la justicia es una virtud artificial debido ante todo a la manera como surge, o bien, debido a la forma oblicua e indirecta en que sirve al interés público y no porque no tenga su fuente en la naturaleza humana ${ }^{9}$. Esta virtud, por tanto, no tiene un carácter espontáneo como la benevolencia sino que siempre conlleva una mediación intelectual. En realidad, nosotros optamos seguir los lineamientos propios de la justicia porque consideramos que seremos favorecidos por las estructuras que ésta genera. Se trata, pues, de una virtud cuya práctica responde siempre a circunstancias materiales concretas y que, por ese mismo motivo, no existe ni puede existir un principio de índole a priori al cual podamos remitirnos para ejercerla ${ }^{10}$.

${ }^{9}$ Barry STROUD, Hume, trad. Antonio Zirión, México: UNAM 2005, p. 286.

10 Resulta evidente el interés por recuperar el enfoque humeano de la justicia de cara a la resolución de problemas concretos. A juicio de estos críticos, una aproximación de este tipo 
Para ilustrar esta aseveración, Hume propone realizar cuatro ejercicios imaginarios - algo muy parecido a lo que en la filosofía analítica se conoce como thought experiments - para probar la veracidad del carácter artificial y utilitario de la justicia. Los expondré de manera concisa para así poder discutir lo que se sigue de ellos:

\section{La superabundancia de los bienes}

En primer lugar, el filósofo escocés sugiere imaginarnos un estado de cosas en el cual la naturaleza haya proporcionado a todos los individuos una superabundancia de recursos de tal suerte que nadie tuviese el más mínimo deseo de privar a otro individuo de sus posesiones. Estos individuos hallarían todos sus apetitos saciados y no se verían sujetos nunca a la necesidad ni a la precariedad, ni tendrían que hacer esfuerzo alguno para su manutención o supervivencia.

\section{La generosidad y la amistad innatas}

El segundo ejercicio es imaginarnos una sociedad, con las mismas características y necesidades que la nuestra, pero que contara con ciudadanos cuyas únicas motivaciones para actuar fueran la amistad y la generosidad. En virtud de tal benevolencia omniabarcante resulta claro que la aplicación de la justicia sería superflua e innecesaria y que los conceptos de propiedad y obligación carecerían igualmente de sentido. No habría necesidad de obligar a nadie a cumplir con un contrato o una promesa que nos beneficiaría pues nadie tendría la inclinación de realizar lo contrario.

\section{El estado de absoluta precariedad}

En un tercer momento, Hume propone imaginarnos una sociedad

nos permite evaluar desde una mejor perspectiva los problemas sociales que la aproximación de cuño rawlseano que pretende situarnos como jueces absolutamente imparciales sobre las cuestiones a ser discutidas. A este respecto, la crítica de inspiración humeana de Larry R. Churchill en contra de Rawls es muy elocuente: «El precio de despojarnos de nuestros prejuicios es muy alto, y mucho perdemos en esta operación. Pocos de nosotros estamos dispuestos a considerar como un escenario ideal para las decisiones morales aquel en el cual seamos despojados de todos los aspectos sociales e históricos de nuestras vidas. Tomar decisiones como si fuéramos personas y desinteresadas o seres angélicos no sólo nos parece algo remoto en vistas a nuestras preocupaciones reales, sino que ello parece quitarnos de las mismas herramientas y recursos que hacen que nuestras decisiones sean moralmente correctas», en: Larry R. CHURCHILL, «Looking to Hume for Justice: On the Utility of Hume's View of Justice for American Health Care Reform», en: Journal of Medicine and Philosophy, XXIV-4 (1999), p. 357. 
donde hubiese una gran carencia de bienes materiales. En esta sociedad, ni la frugalidad ni la laboriosidad más grandes podrían evitar que la mayor parte de sus habitantes perecieran ni la extremada miseria del conjunto. En un grado de necesidad tan radical, queda claro que nadie tendría el más mínimo interés por respetar las leyes de la justicia La supervivencia y la autoconservación se vuelven en este caso las máximas bajo las cuales todos rigen su actuar. En situaciones desesperadas como un naufragio, nadie puede esperar que el respeto a la propiedad y a los derechos ajenos sea lo primero que tomemos en consideración para orientar nuestra conducta. Cuando la sociedad está apunto de ser aniquilada, es completamente razonable que «todo hombre provea por sí mismo por todos los medios que la prudencia pueda dictarle o que el sentido de humanidad le permita» ${ }^{11}$.

4. La presencia de un individuo justo en una sociedad criminal El cuarto ejercicio que Hume propone es imaginarnos la conducta de una persona virtuosa en medio de una sociedad de criminales. En un clima lejos de la protección de las leyes y del gobierno, la conducta que esta persona tiene que adoptar si desea sobrevivir es la de emplear todos los medios posibles para asegurar su existencia. Nadie podría recriminarle a una persona así que ejerza la violencia frente a personas malévolas. Lo mismo suele ocurrir en una guerra: adoptar criterios equitativos en el campo de batalla sólo conduciría a la propia destrucción.

En el primer caso, Hume piensa que todas las virtudes sociales serían cultivadas, salvo la justicia, pues queda claro que en una sociedad así tal virtud estaría completamente de sobra. El reparto de los bienes sería innecesario ya que todos contarían con lo suficiente y el concepto de propiedad no tendría que ser instituido pues nadie podría sufrir prejuicio alguno. El filósofo escocés apunta que esto mismo ocurre en el estado actual de la humanidad con los bienes naturales que tienen una abundancia ilimitada como el agua y el aire, pues éstos no son considerados como propiedades individuales de nadie. En consecuencia, nadie puede cometer una injusticia por el uso y el goce de estos bienes. Asimismo, en relación al se- 
gundo caso, Hume dice que sólo en el seno de las familias podemos encontrar una benevolencia semejante. Sólo con familiares o seres muy queridos los límites de la propiedad se difuminan y existe un interés genuino por la felicidad de los otros. De hecho, como nos relata Hume, han existido varias sociedades han querido difuminar los límites de la propiedad y tratar a todos los integrantes como una gran familia, pero el concepto de propiedad siempre tiene que volver a ser reinstituido toda vez que los intereses egoístas de los hombres vuelven a manifestarse ${ }^{12}$.

En los dos últimos ejemplos, en cambio, se deja ver que situaciones de precaria necesidad abolirían las ideas que tradicionalmente tenemos de la justicia. En dichos escenarios, buscar el beneficio personal sería lo moralmente correcto. Se puede inferir de ello que la justicia, entendida en términos de equidad, sería algo completamente absurdo. La justicia tendría que ser entendida como el derecho legítimo a salvaguardar el bienestar personal dentro de un contexto en el cual nadie hace el más mínimo esfuerzo por favorecer a los demás.

En opinión de Hume, las sociedades humanas no incurren por lo general en ninguno de los extremos sugeridos en estos ejemplos. Los seres humanos tenemos la tendencia de buscar nuestro provecho y el de nuestros amigos, pero también somos capaces de llegar a apreciar las ventajas de una conducta más equitativa. La naturaleza nos proporciona bienes, pero para conseguirlos son necesarios el trabajo y la aplicación. Ni la justicia ni la propiedad son, por consiguiente, cosas inherentes a nuestra naturaleza. Son nociones que requieren ser evaluadas en el marco de su utilidad pública en momentos sumamente concretos y específicos. Esto, por supuesto, tiene varias implicaciones. En primer lugar, la concepción de Hume de la justicia anula la ficción de una edad dorada o la de un estado salvaje de naturaleza de naturaleza hobbesiana, pues es claro para él que los gobiernos derivan sus normas e instituyen la justicia a partir de la ambivalencia entre benevolencia y egoísmo que se da entre los hombres. Si existiese un estado de permanente guerra y 
crueldad sería imposible imaginarnos bajo qué condiciones llegaron esos primeros habitantes de la tierra a formular un pacto. Por otro lado, una concepción de la justicia que responde ante todas estas exigencias nos hace pensar que, al menos por fuerzas violentas y de coacción, jamás podremos imponer una equidad perfecta. Es fácil imaginar la tendencia de varias personas a rebelarse contra tal medida cuando sus intereses se ven afectados. Para preservar tales condiciones sería necesario otorgarle un poder excesivo al gobierno, lo cual ciertamente podría volverse muy peligroso.

Con el fin de garantizar la convivencia humana, Hume propone algo que a su parecer resulta sencillo: crear gobiernos con leyes justas y útiles. Según el filósofo escocés, «allí donde los hombres no ceden a una avidez demasiado egoísta o a un entusiasmo excesivo, el sentido común y una ligera experiencia bastan para ese propósito» ${ }^{13}$. De esta forma, basta con un análisis detenido para ver que todo aquello que resulte útil, beneficioso y de interés para el mayor número de personas es aquello que debemos de juzgar como moral. El respeto por los contratos y las promesas, el cuidado de un patrimonio para la familia, el desempeño de una profesión provechosa, entre otras cosas, son todas conductas justas que han de juzgarse como sumamente morales debido al enorme beneficio público que conllevan. De acuerdo con Hume, el carácter abstracto e incluso frívolo de ciertas leyes sólo es aparente, pues si bien la legislación en materias específicas resulta extremadamente convencional, es necesario tomar en cuenta que las mismas sólo tienen como objetivo el beneficio social. Sólo si consideramos esa utilidad pública las leyes pueden ser realmente vinculantes ${ }^{14}$.

${ }^{13}$ HUME, Investigación sobre..., p. 200.

14 En efecto, Hume sugiere que hemos adquirido ciertos hábitos sociales únicamente por su probada efectividad: «La conveniencia o, más bien, la necesidad que conduce a la justicia es tan universal y sugiere tanto en todas partes las mismas reglas, que el hábito toma asiento en todas las sociedades; y no es sin un cierto examen que somos capaces de descubrir su verdadero origen. Sin embargo, la cuestión no es tan oscura, pues, incluso en la vida cotidiana, recurrimos a cada instante al principio de la utilidad pública y preguntamos: ¿En que se convertiría el mundo si prevalecieran tales prácticas? ¿Cómo podría subsistir la sociedad bajo tales desórdenes? Si la distinción o la separación de posesiones fuera completamente inútil, ¿puede alguien concebir que hubiera llegado alguna vez a prevalecer en la sociedad?», en: HUME, 


\section{La génesis y la eficacia de los gobiernos}

Con base en su análisis de la justicia, Hume sostiene que el hombre tiene que legislar en todos los ámbitos donde la naturaleza no lo proveyó de forma suficiente. En este sentido, el gobierno es una instancia sumamente necesaria creada por el hombre, cuyo propósito no es otro que el de velar por los intereses de los ciudadanos. Dado que el hombre busca poseer más bienes de los que podrían satisfacerlo, tiene disposiciones que no son del todo benevolentes y puede ser fácilmente despojado de sus bienes, es necesario que las comunidades adquieran formas y estructuras racionales que permitan solventar todas estas problemáticas. Puede decirse, por consiguiente, que si el gobierno no beneficiara al hombre, éste jamás hubiera sido creado, todos los bienes estarían repartidos de forma equitativa y todas las acciones se realizarían sin perjudicar a los demás ${ }^{15}$. Como de facto nada de eso ocurre, el hombre se ve en la necesidad de autorregular su interacción con los demás individuos por medio de la razón. Es ahí donde se puede apreciar una confluencia entre nuestras virtudes naturales y artificiales: éstas últimas alientan a las primeras y garantizan que puedan llevarse efectivamente a la práctica dentro de una comunidad determinada.

La naturaleza humana no puede subsistir de ningún modo sin la asociación de individuos; y esta asociación nunca podría tener lugar si no se respetaran las leyes de la equidad y la justicia. Como afirma Moore, la clave para entender el pensamiento humeano en este punto es su rechazo a la ficción del estado de naturaleza ${ }^{16}$. Para el filósofo escocés, siempre se saben dependientes los unos de los otros y es por ello que viven en sociedad. El único estado de naturaleza medianamente funcional que podemos concebir es

Investigación sobre..., p. 204.

${ }^{15}$ Churchill hace énfasis en este punto de la doctrina del filósofo escocés: «Como Hume observaba, si la benevolencia fuese un sentimiento tan extendido y tan amplio en su alcance, la necesidad de tener instituciones sería mucho menor de lo que actualmente resulta ser el caso», en: CHURCHILL, «Looking to Hume...», p. 358.

16 Cf. James MOORE, «Hume's Theory of Justice and Property», en: Political Studies XXIV-2 (1976), p. 106. 
la familia ${ }^{17}$, pero de ningún modo una comunidad humana más amplia habría podido subsistir de no ser por que los miembros de ésta encontraran, desde un primer momento, ciertos beneficios inmediatos en la cooperación mutua. La relación inestable que existe entre los seres humanos y sus posesiones tiene como consecuencia que las instituciones políticas hagan su aparición para solventar tal situación de incertidumbre.

Ahora bien, en la doctrina humeana, las posiciones que cifran la génesis del gobierno en un contrato social original pecan de ser terriblemente ingenuas. Tanto en sus obras principales como en el ensayo donde aborda concretamente ese tema ${ }^{18}$, Hume despliega una serie de críticas muy duras en contra del contractualismo. Los argumentos que esgrime en su opúsculo «Sobre el contrato original» son la mayor parte de las veces históricos o se remiten a experiencias concretas. Dice Hume, por ejemplo, que los principios que guían la cooperación de los seres humanos rara vez se explicitan desde un principio: «el consentimiento y las ventajas resultantes de la paz y el orden ${ }^{19}$ pueden darse sin que un contrato muestre tales beneficios de forma explícita. En el mismo tenor, Hume hace ver que la mayoría de los gobiernos rara vez se fundan en un acuerdo: lo que tiende a ocurrir, más bien, es que el poder se constituye a partir de guerras o invasiones que terminan consolidando a un Estado. Los incesantes cambios en los asuntos humanos, la sucesión ininterrumpida de generaciones a través de los años, y las condiciones apremiantes a las que cada comunidad se enfrenta, hacen completamente imposible pensar que la legitimidad de un gobierno descanse en el respeto que los ciudadanos puedan sentir por un pacto realizado décadas o siglos atrás. A este respecto, Hume propone que realicemos otro ejercicio imaginativo: si pensamos,

17 La familia tiene tres debilidades básicas que, a juicio de Moore, hacen que la cooperación social se dé prácticamente de forma inmediata: «la debilidad de los seres humanos en relación a otras criaturas y elementos; la falta de astucia y de habilidades económicas especiales; y la tercera y más importante: la inseguridad en las relaciones interpersonales», en: MOORE, «Hume's Theory...», p. 106.

18 Cf. David HUME, «Of the Original Contract», en: Selected Essays, Oxford: Oxford University Press 1998, 274-292 pp.

19 HUME, «Of the Original...», p. 276. 
por ejemplo, el caso de un príncipe que es depuesto en el trono por otro, pero que regresa después de muchos años con un ejército mayor y recuperara el poder, ¿no sería absurdo suponer que lo que legitima su poder es el consentimiento de la gente? Por el contrario, «ellos consienten porque lo consideran, por nacimiento, su legítimo soberano» ${ }^{20}$. La tradición jugaría en ese caso un papel determinante y un asentimiento explícito sería completamente innecesario. Sin embargo, también podemos imaginarnos situaciones en las que la tradición, por más entrañable que sea para una nación, no jugaría ningún papel: si hoy en día se presentara el legítimo descendiente de los emperadores aztecas reclamando el trono de México, sería muy poco probable que su abolengo fuese un motivo suficiente para que la población hiciera de lado las instituciones por las cuales se han regido los pasados siglos. La cuestión, como se ve, resulta ser movediza, tal y como lo es la aplicación de la justicia en los distintos escenarios a los que podemos enfrentarnos.

Por esta razón, Hume afirma que es pueril la situación de libertad en la cual Hobbes y Locke se imaginan tanto a los realizadores del pacto como a aquellos que hoy en día supuestamente los respetan. La pregunta que arroja frente a sus contrincantes, en este sentido, es interesante: «¿Podemos decir, seriamente, que un pobre campesino o artesano es libre de abandonar su país, siendo que no conoce el lenguaje ni las costumbres extranjeras y vive, día a día, de los pequeños sueldos que recibe?» ${ }^{21}$. A todas luces, éste es un caso en el cual habría pocos argumentos para afirmar que dicha persona observa los lineamientos de un gobierno por respeto al contrato original.

Hume, por supuesto, también tiene el interés de defender la cooperación social desde una perspectiva más filosófica. Cito en extenso un pasaje clave del Tratado:

[La convención que da origen a la sociedad] es sólo un sentimiento general del interés común, que se comunican unos a otros todos los miembros de la sociedad y que los induce a regular su conducta me-

${ }^{20}$ HUME, «Of the Original...», p. 285.

${ }^{21}$ HUME, «Of the Original...», p. 285. 
diante ciertas reglas. Me doy cuenta de que favorecerá mi interés dejar a otro en la posesión de sus bienes, dando por sentado que él actuará conmigo de la misma manera. En la regulación de su conducta, él puede percibir un interés semejante. Cuando esta percepción común del interés se comunica mutuamente y resulta conocida de ambos, produce la resolución y la conducta adecuadas. Y esto puede ser llamado con suficiente propiedad una convención o acuerdo entre nosotros, aun sin la interposición de una promesa; pues las acciones de cada uno de nosotros tienen una referencia a las del otro y se llevan a cabo en la suposición de que la otra parte también llevará algo a cabo. Dos hombres que impulsan un bote a fuerza de remos, lo hacen en virtud de un acuerdo o convención, aunque nunca se hayan prometido nada el uno al otro ${ }^{22}$.

Es evidente, a juicio de Hume, que la cooperación debe pensarse en términos de ventajas recíprocas y que sólo el reconocimiento de las mismas puede garantizar la participación de los agentes en aras de los mayores beneficios. Las promesas, por su propia naturaleza, no son más que resoluciones acompañadas de ciertas palabras. Pero, como bien destaca Moore, esas palabras carecerían de todo sentido «si los hombres no hubiesen aprendido a regular su conducta y su lenguaje de acuerdo a reglas más o menos generales» ${ }^{23}$. De esto se sigue que las reglas y los juicios sobre las cuales estás se basan son anteriores a los contratos y a las promesas mismas. Por ello, en opinión de Hume, es completamente falaz fundamentar la existencia de reglas y los juicios que las justifican en los contratos y las promesas.

De forma perspicaz, Hume intenta, por una parte, que la consolidación de los distintos gobiernos a lo largo de la historia no se encuentra basada en el acuerdo explícito por parte de los miembros de su comunidad y, por otra parte, que la vigilancia de las leyes de un gobierno se dé en virtud del respeto que tenemos por el contrato original. En su opinión, más bien, somos capaces de encontrar beneficios en la cooperación incluso desde un momento anterior a 
que ésta sea enunciada de forma explícita en una especie de pacto. Más aún, somos agentes que de alguna manera siempre velamos por nuestros intereses desde la perspectiva de una racionalidad estratégica, y es por ese motivo que consideramos valiosos los acuerdos que conducen a un mayor beneficio para la mayor cantidad de integrantes de una sociedad. Sólo esa clase de mayor beneficio para todos reditúa en que yo, como ciudadano particular, obtenga de manera oblicua o indirecta más beneficios a la larga. El mismo carácter artificial de las leyes permite que, en cualquier momento o circunstancia en que éstas sean ineficientes, nosotros seamos capaces de crear nuevas convenciones que tengan como propósito proporcionar mayores ventajas a toda una comunidad.

A la luz de lo anterior, me parece importante realizar unas breves conclusiones. Pienso que la teoría humeana de la justicia tiene una gran relevancia contemporánea. Considero que la forma pragmática y dinámica en la que concibe los procesos políticos y la articulación de leyes permite dar cuenta de cómo es posible que se den ciertas transformaciones sociales desde una posición que, si bien escapa a las limitadas perspectivas de un utilitarismo burdo, permite articular de buena forma la intuición moral básica de que todos buscamos obtener el mayor provecho posible de las instituciones que formamos. Por supuesto, la posición de Hume enfrenta también muchas reservas. Me viene a la mente, por ejemplo, en la crítica de Barry Stroud. De acuerdo con Stroud, si bien Hume argumenta de forma sólida que los ciudadanos respetan las convenciones de la justicia y de la propiedad porque quebrantarlas implicaría destruir la sociedad, el filósofo escocés no tendría elementos para censurar a un individuo que quiera cometer una pequeña injusticia que no rompa todo el orden social ${ }^{24}$. Si una persona comete una pequeña injusticia - que no destruye la estabilidad del gobierno - en aras de obtener un beneficio personal, Hume no podría censurar dicha conducta porque su énfasis en la conducta interesada de las personas no tendría los suficientes elementos normativos.

En mi opinión, sin embargo, la fuerza de la teoría humeana radica justo en que puede invocar en esos casos el interés personal de 
los agentes racionales. La crítica de Stroud concibe a un agente que opera única y exclusivamente mediante una racionalidad paramétrica: en otras palabras, se considera a sí mismo el único que rompe un sistema de reglas ${ }^{25}$. Pero no toma en cuenta que estas pequeñas injusticias imperceptibles a la larga alterarían inevitablemente el orden social. Una respuesta humeana, en este sentido, iría enfocada a mostrar que en realidad cometer esa pequeña injusticia atentaría contra mi propio beneficio al largo plazo. Ante estas críticas, Hume no necesitaría cambiar en lo más mínimo su postura en relación al interés personal. Algo también importante es que resulta una teoría que no intenta apelar a modelos ahistóricos de normatividad que no contemplan las formas distintas en las que la justicia puede ser concebida. En todo caso, considero cuestionable la forma en que Hume articula su propuesta moral sin tener en cuenta la perfección que la práctica de la virtud brinda a quien la realiza, pero no me parece que se trate de una crítica que pueda trasladarse a su teoría de las instituciones sociales y políticas.

${ }^{25}$ Desarrollo esta crítica a Stroud basándome en las ideas generales del capítulo «La teoría de la elección racional» de Elisabetta DI CASTRO, La razón desencantada. Un acercamiento a la teoría de la elección racional, México: UNAM 2002. Tengo la impresión de que este problema bien podría ser enfocado desde la teoría de juegos. Espero poder desarrollarlo más a fondo en una próxima investigación. 


\section{RESUMEN}

Este artículo revisa los presupuestos fundamentales del pensamiento político humeano. En él se analiza cómo es que, a juicio del filósofo escocés, razonamos los seres humanos en términos de justicia y por qué es necesario crear instituciones que coordinen estratégicamente los intereses de una comunidad. Finalmente, se estudia la crítica humeana al contractualismo imperante de su época.

Palabras clave: Hume; virtudes artificiales; filosofía política; contractualismo.

\section{ABSTRACT}

This paper discusses the fundamental assumptions of the Humean political thought. It analyzes how, according to the Scottish philosopher, we reason in terms of justices and why it is necessary to create institutions that strategically coordinate the interests of a community. Finally, it studies the Humean critic against the contractualism of his times.

Key words: Hume; artificial virtues; political philosophy; contractualism. 